Situación de la educación universitaria en el contexto de la consolidación de la cultura democrática Fernandes, O. A. y el desarrollo social en El Salvador

\title{
Situación de la Educación Universitaria en el contexto de la consolidación de la cultura democrática y el desarrollo social en El Salvador
}

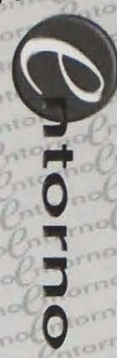

Poh: Óscar A. Firnánidzz

José Miguli. AMAYA

$\mathrm{L}$ os años de 1979 y 1980 fueron el comienzo del conflicto armado y de la casi inmovilidad gubernamertal, a lo quese agrega la salida masiva de capitales, las grandes concentraciones populares, los asesinatos, secuestros, ametrallamientos, bombas y otras sictuaciones dolorosas. En estos dos años, no hubo nuevas universidades y se produjo el cierre e intervención militar de la Universidad de El Salvador (UES) en 1981

En este periodo, se aumentó la demancla por nuevas universidades, presión que se liberó en ese mismo aiio, cuando muchos acadénicos y empresarios interesados crearon universidades con el beneplácito y la promoción gubernamental. El 12 de junio de 1981 fue aprobada por Decrero Ejecurivo la Universidad Tecnológica de El Salvador. Diez nuevas universidades surgieron con enfoques, orientaciones y modelos innovadores, tales como las universidades tecnológicas, las univer sidades humanísricas, las de carácrer religioso, las especializadas en el área de salud y las universidades descentralizadas o desconcentradas de San Salvador.

Se abren nuevas carreras consideradas como tabú, tales como: mercadeo, comunicaciones, computación, diseño ambiental, diseño gráfico; asimismo, las macstrias profesionales $y$ las carreras en trabajo social, educación especializada en adultos, educación parvularia, currículo, educación especial, educación en población, relaciones públicas, además de diversos profesorados en aspectos importantes, como orientación educariva, evaluación escolar, Agronomia, Idioma Inglés y otras carreras técnicas necesarias para el desarrollo del pais y enfocadas a diversidad de objetivos educativos. A principios de los noventa, más de cuarenta instituciones de educación superior funcionaban en El Salvador.

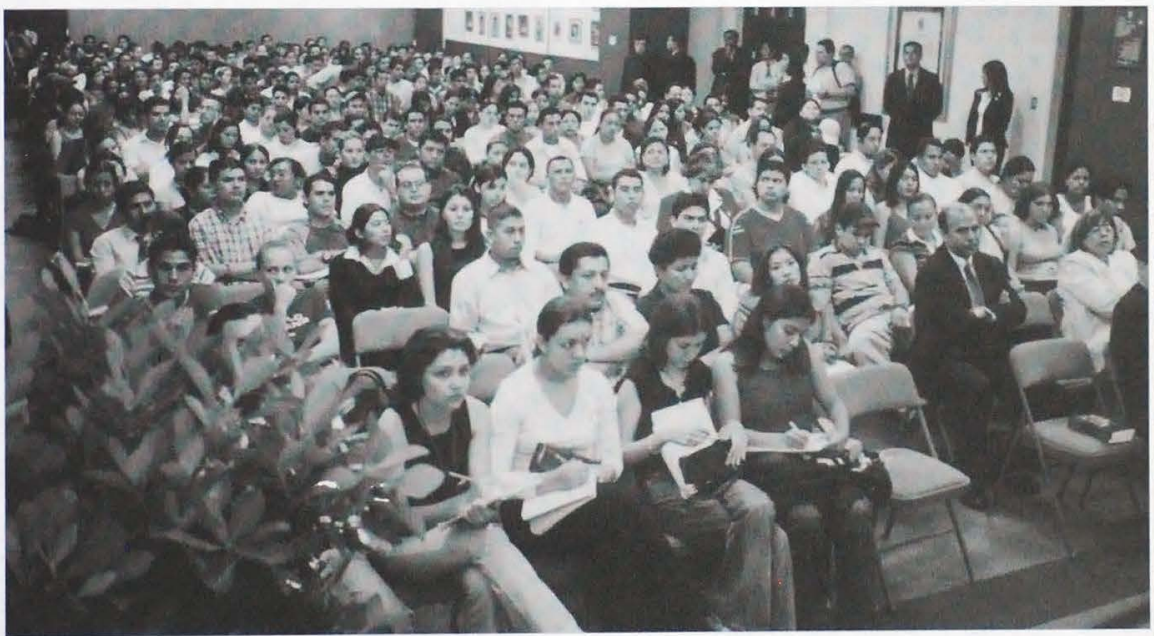




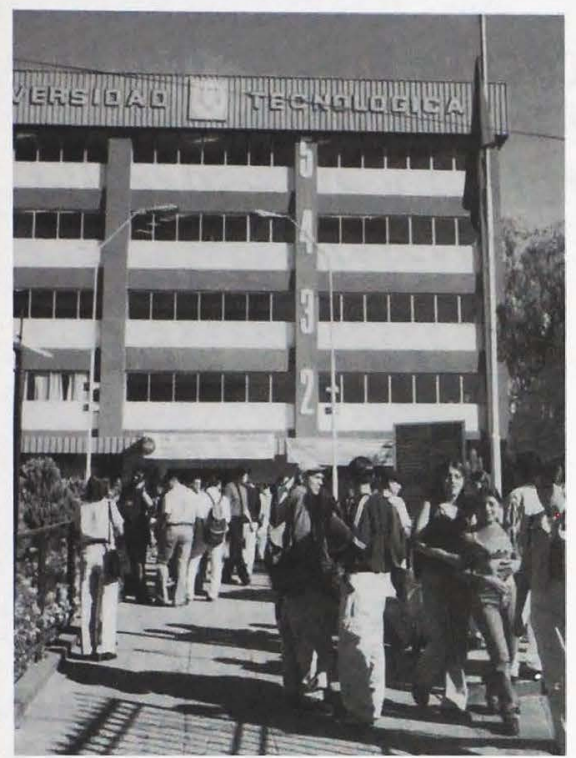

La actual Ley de Educación Superior fue aprobada el 30 de noviembre de 1995, mediante Decreto Legislativo 522 y se publicó en el Diario Oficial el 20 de diciembre de 1995. Posteriormente, se elaboró el reglamento general con fecha de 9 de agosto de 1996. La nueva ley estableció un plazo especial de dos años para que las instituciones se adecuaran a las exigencias de la misma.

Entre los aspectos legales contenidos en la ley, que más han incidido positiva y negativamente en el funcionamiento de las instituciones de educación superior (IES) se rienen los siguientes: decerminación de las unidades valorativas (UV), cuyo valor por unidad equivale a 20 horas de trabajo del estudiante atendidas por un profesor. Relación de un profesor a tiempo completo por cada 75 estudiantes, $y$ un profesor por cada 35 estudiantes.

1)uración de la hora clase de 50 minutos. Definición de grados académicos y número de unidades valorativas por cada grado, así como la duración de las carreras, establecimiento del coeficiente de unidades de mérito (CUM) como el cociente resultante de dividir el total de unidades de mérito (calificación final de cada materia multiplicada por sus unidades valorativas) ganadas, entre el total de unidades valorativas de las asignaturas cursadas y aprobadas.

Para crear una nueva carrera, se requiere un estudio de facribilidad y demanda, al igual que para crear una nueva institución y/o descentralizar el funcionamiento de las instituciones. Se creó el Consejo de Educación Superior (CES), definido como órgano consultivo y propositivo del Ministerio de Educación (MI NEI)), el cual está conformado por: a) Dos representantes del MINED. b) Un representante de la UES. c) Tres representantes de las universidades privadas, d) Un representante de los institutos tecnológicos. e) Un representante de las asociaciones gremiales de la empresa privada $y, f)$ Un representante de las asociaciones gremiales de prof esionales.

También se creó en la misma ley cl Sistema de Supervisión y Mejoramiento de la Calidad de la Educación Superior en El Salvador, el cual contempla Subsistemas de calificación, evaluación y acreditación. Tanto la calificación como la evaluación las administra el MINED y son obligatorias, y la acreditación, que es voluntaria, es aplicada por una Comisión de Acreditación, la cual está integrada por académicos de notoria capacidad y honradez, quienes no representan a ninguna institución y son nombrados de mutuo acuerdo entre el MINED y el (CES) para un periodo de cuatro años. La calificación es eminentemente cuantitativa y está referida a la estimación de ratios de carácter cuantitativo tales como: número de estudiantes por docente, porcentaje de docentes a tiempo completo, número de libros por estudiante, número de estudiantes por computadora, espacio académico por estudiante. espacios para recreación, costo por estudiante.

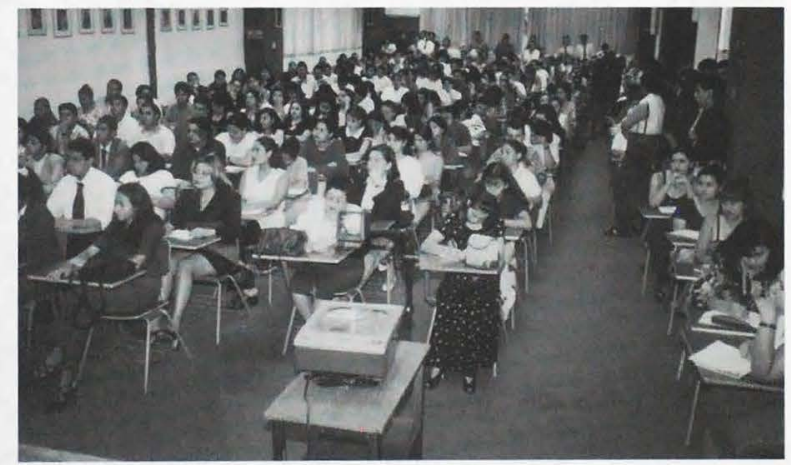


La evaluación es cualitaciva y sefundamenta en un estudio de auroevaluación institucional y la posterior verificación de pares excernos, que producen un inf orme oral y escrito el cual es remitido a la institución evaluada y ésta, a su vez, reacciona aceprando o rebaciendo con pruebas las observaciones ante el MINED, para que éste posteriormente se pronuncie en una resolución ministerial, que requiere la aprobación previa del CES, y que las instiruciones deben de acatar.

Los criterios para la realización de la autoevaluación son los siguientes: 1) La misión institucional. 2) El gobierno y administración institucional. 3) Estudiantes. 4) Académicos. 5) Carreras y otros programas. 6) Investigación. 7) Proyección social. 8) Recursos educacionales. 9) Administración financiera. 10) Infraestructura y 11) Integridad inscitucional.

En la actualidad, existen 42 instituciones de educación superior entre universidades, instituros tecnológicos e instituciones especializadas. Más de 114.675 estudiantes están macriculados en las diferences universidades pública y privadas, correspondiendo un $72 \%$ de esa población a la participación de la educación superior privada.

Debe señalarse, sin embargo, que la ley tiene también vacíos e insuficiencias, las cuales se espera superarmediante un cambio en la ley promovido por los diversos actores involucrados incluyendo al CES, $y$ al Foro Permanente de Universidades que compuesto por universidades de pres-

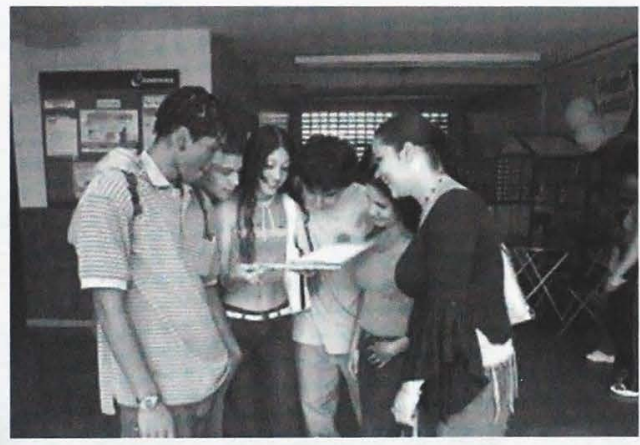
rigio y a la Universidad de El Salvador, además de otras asociaciones de universidades, tales como AUPRIDES, que cuenta con ocho universidades de reconocida trayectoria en el pais.

Entre las falencias y debilidades obvias de la LES se pueden mencionar las siguientes: es una ley creada para cerrar instituciones y castigar malas actuaciones; no es una ley incentivadora. Fue creada para ordenar el desorden, más que para enunciar normas orientadoras. Carece de definiciones conceptuales profundas en sus declaraciones y tiene un reglamento muy general que no operacionaliza los diversos procedimientos de la ley que requieren las IES.
Establece algunos parámetros cuantitativos, que no son aplicables ante el avance de la ciencia y la tecnología, tales como número de alumnos por prof esor, duración de la hora clase, obsesión por los espacios. Restringe la innovación y creación de carreras y centros descentralizados por procedimientos burocráticos más de forma que de contenidos.

Deja vacíos enormes, al no contemplar nada sobreeducación a distancia, educación virtual y educación continua, las cuales deberian de tener claras orientaciones e incentivos. La obligatoriedad de cumplir con tres funciones universicarias, que en la actualidad han sido superadas en muchos paises como son la docencia, la proyección social y la investigación, no sólo diluyen recursos, sino que ademásgeneranproblemas serios de organización interna en las IES.

Esto que bien podria regularse en función de las visiones y misiones univer sitarias, de los recursos e incentivos estatales y sobre rodo, de la mulcifuncionalidad institucional e integración de focos de canalización de prioridades, es todavía algo que se pretende estandarizar sin razones técnicas y mucho menos fácricas.

Los cambios más importantes que se contemplan en el proyecto de reforma a la LES, aprobado y propuesro por el Consejo de Educación Superior (CES), seorientan a los siguienres tópicos: Incluir aspectos orientadores de carácter estratégico para el país, acrualizar concepros fundamentales para la educación y definitlos más, especificación de limitantes para lacreación de nuevas instituciones, regulación de IES exrranjeras, modificación de la cantidad de profesores en relación con el número de estudiantes ampliándola a un prof esor por cada cuarenta estudiantes $y$, de esa relación, que sean profesores a tiempo completo un 25 porciento.

También incluye la creación de un reglamento especial para educación a distancia, creación de una unidad de vigilancia e inspección de IES, ampliación del riempo para la evaluación de las IES de dos a cada tres ańos, incegración de los procesos de evaluación y acredicación, y una mayor cancidad de especificación de detalles de liquidación y disolución de IES. 


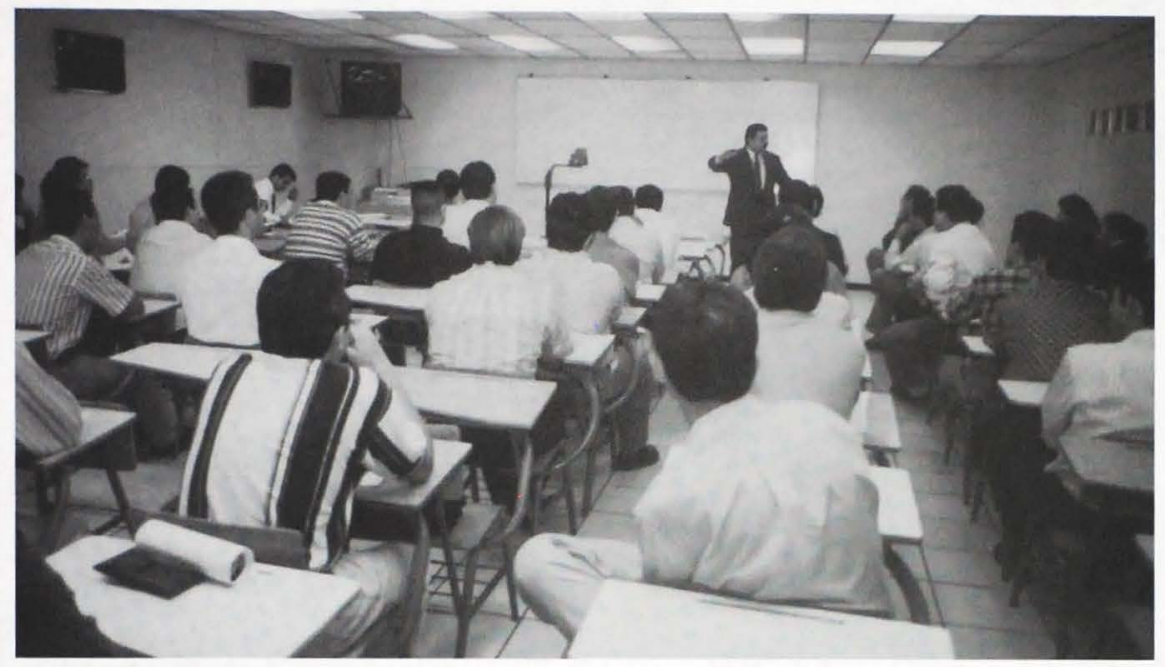

La ley vigente de educación superior atenúa algunos aspectos que asfixian a las IES, aunque mantiene una condición reguladora y fiscalizadora, en contraposición con una ley que debería brindar más incentivos a aquellas IES que tienen credibilidad en sus procesos y que evidencian mayores esfuerzos por innovar y mejorar la calidad académica.

Se necesita una ley que oriente, norme y def ina claramence una nueva visión para la educación superior, de conformidad con los riempos presentes y fururos, y que enuncie una misión recadora, creativa e inspiradora de nuevos compromisos, para el nuevo mundo que invade a nuestras sociedades.

La investigación ha tenido como objecivo presentar una explicación situacional y causal del desarrollo de la enseñanza universitaria, el rol desempeñado por las universidades en el desarrollo del ser humano, su aporte a la sociedad democrática que deseamos y la accitud del Estado al respecto, con el propósito de diseiniar las bases de un plan nacional de la educación superior que profundice la calidad y amplie la capacidad de cobertura de la demanda por formación universitaria en el pais en los próximos dię años.
Los propósito han sido dos fundamentalmente:

I. Presentar los resultados a los parcidos políticos que han contendido por la presidencia del Poder Ejecurivoy, desde luego, al nuevo gobierno que resultó electo.

2. Desarrollar un proceso Estado-universidades, para diseñar las bases de un plan nacional de educación superior, que prof undice la calidad y amplíe la capacidad de cobertura

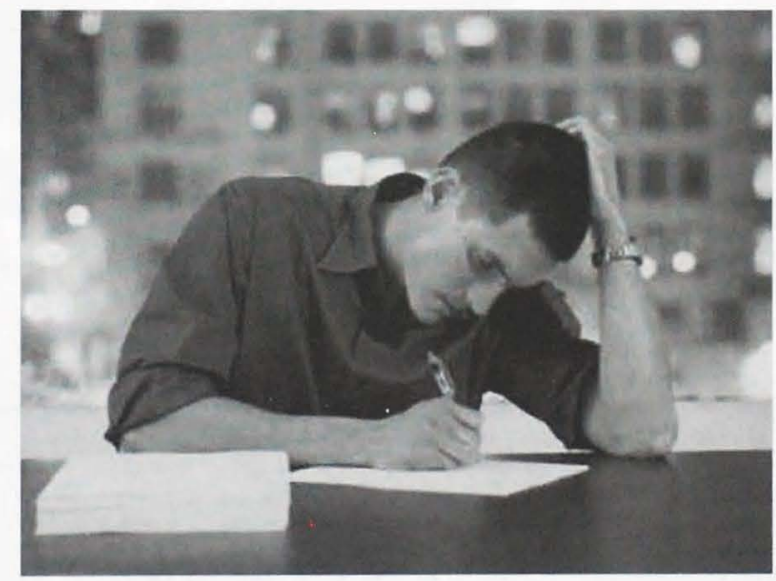

Entorno

ISSN: 2218-3345 
a la alca demanda de formación universitaria en el país, para los próximos diez años.

El problema de investigación se definió asi: "Relación entre la calidad de la formación universitaria y la voluntad y magnitud del soporte político y económico del Estado salvadoreño, en el contexto del proceso de construcción y consolidación de la cultura democrática y el desarrollo integral del país (1992-2004)".

Se emplearon los métodos hipotético deductivos para describir el problema, y el de análisis inductivopara explicar el desarrollo histórico (acumulaciones) y la estructura causal (genoestructura) de dichos resultados o descriptores del problema.

La investigación se centró en la recolección de datos pertinentes fundamentalmente a través de entrevistas con personas especializadas $y$ directamente vinculadas al sistema educativo de formación universitaria (decanos, directores académicos, diputados, funcionarios públicos del ramo y expertos académicos relacionados con la problemática universitaria nacional), asicómo de documentos a d hoc, leyes y reglamentos de la nación, anteriores investigaciones acreditadas, leyes especiales del presupuesto nacional, documentos de evaluación y resultados del MINED y bibliografía científica especializada.

El estudio consta de los siguientes componentes: Revisión de los elementos pertinentes que estructuran el problema. Formulación de un sistema de hipótesis. Recopilación de los datos. Descripción y explicación del problema investigado. Diseño de un modelo teórico que explique el problema. Diseño de un modelo lógico de cómo abordar el problema en la búsqueda de sus soluciones más importantes y urgentes. (Recomendaciones).

Explicación de los componentes más relevantes del problema -nudos críticos-

1. Las universidades privadas no son exigentes en la escogitación y desarrollo de alumnos y profesores, ya que dependen en un $90 \%$ de los ingresos por matrículas y escolaridades.

2. No existe soporte económico alguno del Estado a las Universidades privadas y el presupuesto de la universidad estatal es deficitario.

3. Las herramientas bibliográficas, documentales y tecnológicas son insuficientes frente a la nueva demanda de la modernización.

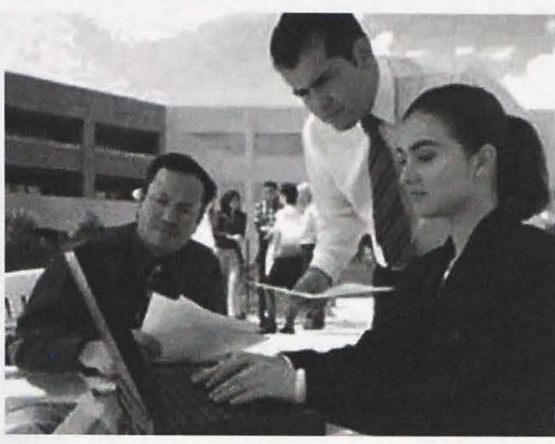

4. La investigación cientifica de alto nivel académico, en general no es un requisito en la formación académica universitaria.

5. La normativa legal no es especifica en cuanto a homologación de los procesos, controles y conceptos de calidad, permitiendo un amplio margen de discreción en la interpretación

6. Las políticas públicas y presupuestarias a favor del desarrollo de la educación superior universitaria en la última década, no aparecen como una prioridad.

7. E! Estado no ha desarrollado el apoyo necesario para generar una cultura de investigación ciencífica y las universidades la realizan marginalmente.

8. No existen programas científicos, sistemáticos y ef icaces para desarrollar, a través de la educación, la formación de la cultura democrática.

9. La enseñanza media produce bachilleres de baja calidad académica.

10. La educación en general y la universitaria en especial. no se demuestra en el presupuesto de la nación y en la acción del Estado, como el fundamento del desarrollo nacional.

11. La formación académica y científica no aparecen como un rasgo clásico y fundamental de nuestra cultura educativa.

Las recomendaciones del estudio planteado son las siguientes:

1. Las universidades deberían de manera conjunta, revisar exhaustivamente los estándares y procedimientos de 
selección estudiantil y docente, procurando el soporte económico del Estado, con el propósito de financiar cursos de nivelación y propedéuticos para alumnos y de profesionalización para los docentes. Se recomienda que se haga similar gestión ance la cooperación internacional.

2. Las universidades acreditadas (privadas y estatal) deben de manera conjunta, construir y presentar una propuesta lógica y racional que implique el compromiso legal del Estado de asignar para la educación superior universitaria, un porcentaje mayor del presupuesto de la nación en relación con el producto interior bruto (PIB), tomando de base lo

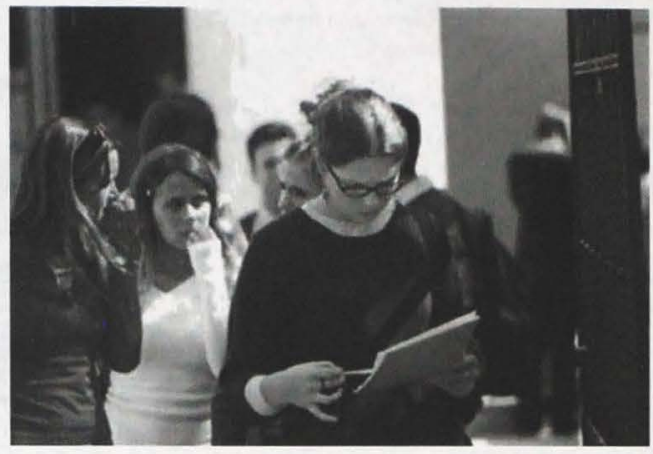
asegurado en los Arts. 53, Inc. $1^{0} ; 57$, Inc. $2^{\circ} ; 61$, Inc. $3^{\circ} \mathrm{C}$.

3. Las universidades acreditadas deberían establecer mecanismos de corto plazo para estandarizar las bibliotecas y la tecnología avanzada, necesarias para el desarrollo básico de la currícula y los programas de estudio. Es necesario buscar apoyo conjunto con las organizaciones internacionales multi laterales dedicadas a la rama de la ciencia y la cultura.

4. Que se establezca normativamente que el desarrollo de la investigación y la calidad de producción científica, se constituyan como dos parámetros fundamentales para la acreditación univer sitaria. De igual forma, sus avances signifiquen un requisito para la obtención de estímulos y premios que el Estado deberá otorgar a las Universidades.

5. Después de la experiencia transcurrida, la LES y el reglamento respectivo, deben revisarse en el sentido de delimitar y especificar el sistema de control, supervisión y acreditación de las universidades, con el propósito de coad yuvar al desarrollo cualitativo de lasmismasy lograr un consenso positivo con el Estado.

6. Es necesario que las universidades acreditadas participen en la discusión de la elaboración de las partidas presupuestarias de educación, especificamente en el rubro de educación superior. Asimismo, deben lograr, a través de la gestión correspondiente, que la Asamblea Legislativa escuche a los rectores cada vez que se discuta esta área presupuestaria en la Comisión de Hacienda y Presupuesto de la Asamblea Legislativa.

7. Que el Estado se compenerre de su rol como ente principal para el desarrollo de la investigación y el

8. Las universidades deberian elaborar una propuesta de plan para desarrollar el estudio sistemático de derechos humanos, civismo, solidaridad y democracia, que pueda ser impartido regularmente desde la primaria. En el proceso, se recomienda buscar el aval de las autoridades gubernamentales $y$ los partidos políticos.

9. Que se demande a las autoridades de Educación y a la Comisión de Educación de la Asamblea Legislativa, el abordaje inmediato del problema de la baja calidad de bachilleres que en general se están graduando y pretenden ingresar a las universidades. Que en la búsqueda de soluciones se involucre, tanto al nivel de bachillerato como a las universidades.

10. Que el Estado debe comprometerse ha establecer como segunda prioridad de la nación, después de la salud, a la educación, y dentro de ella, reconocer que la formación universitaria es estratégica para el desarrollo del conocimiento cientifico y tecnológico y el avance del país.

11. Que para impulsar un nuevo y más influyente sistema de educación nacional, es necesario comenzar de inmediato a forjar una cultura social con aspiración educativa y lograr que a la base de nuestro desarrollo se logre ubicar la concepción científica de la realidad, como la base para lograr la plena realización de los seres humanos. 